\title{
2021 CAEP Acute Atrial Fibrillation/Flutter Best Practices Checklist
}

\author{
Ian G. Stiell ${ }^{1,2,23}$ - Kerstin de Wit ${ }^{4,5} \cdot$ Frank X. Scheuermeyer $^{3} \cdot$ Alain Vadeboncoeur $^{11,12} \cdot$ Paul Angaran $^{18}$. \\ Debra Eagles ${ }^{1,2} \cdot$ Ian D. Graham ${ }^{2}$. Clare L. Atzema ${ }^{7,8} \cdot$ Patrick M. Archambault $^{10} \cdot$ Troy Tebbenham $^{14}$. \\ Andrew D. McRae ${ }^{9}$. Warren J. Cheung ${ }^{1,2} \cdot$ Ratika Parkash $^{19} \cdot$ Marc W. Deyell $^{20} \cdot$ Geneviève Baril $^{13} \cdot$ Rick Mann $^{15}$.

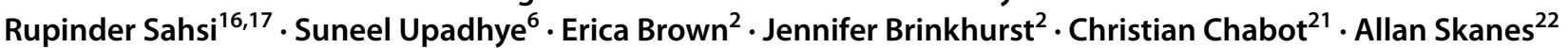

\section{A. Assessment and risk stratification}

\section{Is AF/AFL with rapid ventricular response a primary arrhythmia or secondary to medical causes?}

A. Rapid rate secondary to medical causes (usually in patients with pre-existing/permanent AF) e.g., sepsis, bleeding, PE, heart failure, ACS, etc.:

- Investigate and treat underlying causes aggressively

- Cardioversion may be harmful

- Avoid aggressive rate control

Ian G. Stiell

istiell@ohri.ca

1 Department of Emergency Medicine, University of Ottawa, Ottawa, ON, Canada

2 Ottawa Hospital Research Institute, Ottawa, ON, Canada

3 Department of Emergency Medicine, University of British Columbia, Vancouver, BC, Canada

4 Department of Emergency Medicine, Queen's University, Kingston, ON, Canada

5 Department of Medicine, McMaster University, Hamilton, ON, Canada

6 Division of Emergency Medicine, McMaster University, Hamilton, ON, Canada

7 Division of Emergency Medicine, University of Toronto, Toronto, ON, Canada

8 Institute for Clinical Evaluative Sciences, Toronto, ON, Canada

9 Department of Emergency Medicine, University of Calgary, Calgary, AB, Canada

10 Department of Family Medicine and Emergency Medicine, Université Laval, Quebec, QC, Canada

11 Université de Montréal, Montreal, QC, Canada
B. Primary arrhythmia, e.g., sudden onset of AF/AFL

TIP: Rapid rate is more likely to be secondary to an underlying medical cause if:

- Not sudden onset, no palpitations

- Known permanent $A F$, on OACs, old ECGs show $A F$

- No history of ED cardioversions

- $H R<150$

- Fever, dyspnea, pain
12 Department of Emergency Medicine, Montreal Heart Institute, Montreal, QC, Canada

13 Hôpital de Granby, Granby, QC, Canada

14 Peterborough Regional Health Centre, Peterborough, ON, Canada

15 Trillium Health Partners, Mississauga Hospital, Mississauga, ON, Canada

16 Division of Emergency Medicine, Department of Family Medicine, McMaster University, Hamilton, ON, Canada

17 St. Mary's General Hospital, Kitchener, ON, Canada

18 Division of Cardiology, Terrence Donnelly Heart Centre, St Michael's Hospital, University of Toronto, Toronto, ON, Canada

19 Division of Cardiology, Dalhousie University, Halifax, NS, Canada

20 Heart Rhythm Services, Division of Cardiology, University of British Columbia, Vancouver, BC, Canada

21 Quebec City, QC, Canada

22 Division of Cardiology, Western University, London, ON, Canada

23 Clinical Epidemiology Unit, The Ottawa Hospital, F657, 1053 Carling Avenue, Ottawa, ON K1Y 4E9, Canada 


\section{Is the patient unstable?}

- Instability due to acute primary AF/AFL is uncommon, except for AF with rapid ventricular pre-excitation (WPW):

- Hypotension: $\mathrm{SBP}<90 \mathrm{mmHg}$, or signs of shock (e.g., altered mental status)

- Cardiac ischemia: ongoing severe chest pain or marked ST depression (>2 mm) on ECG despite therapy

- Pulmonary edema: significant dyspnea, crackles, and hypoxia

- Treat unstable patient:

- Urgent electrical CV if onset $<48 \mathrm{~h}$ or WPW

- Consider trial of rate control if onset $>48 \mathrm{~h}$

\section{Is it safe to cardiovert this patient with primary AF/AFL?}

- When it is safe, rhythm control is usually preferable to rate control: patient quality of life, shorter length of stay, fewer hospital resources

- It is safe to cardiovert if:

A. The patient has been adequately anticoagulated for a minimum of 3 weeks, OR

B. The patient is not adequately anticoagulated for $>3$ weeks, has no history of stroke or TIA, AND does not have valvular heart disease, AND:

1. Onset $<12 \mathrm{~h}$ ago, OR

2. Onset $12-48 \mathrm{~h}$ ago and there are $<2$ of these CHADS- 65 criteria (age $\geq 65$, diabetes, hypertension, heart failure), OR

3. Negative for thrombus on transesophageal echocardiography

- Consider delaying cardioversion if recent history of frequent palpitations

- Rate control acceptable, per patient and physician preference

- e.g. older patients who are minimally symptomatic with a mildly elevated HR
TIP: How to determine if therapeutic OAC $\times 3$ weeks?

Based on MD judgment

DOAC - confirm compliance by history

Warfarin

- Current INR $>2.0$

- Recent INR values $>2.0$

- Recent INR testing confirmed by history

- No recent changes in dose

\section{B. Rate and rhythm control}

\section{Rate control for patients for whom cardioversion is unsafe}

- Calcium channel- and beta-blockers considered first line:

- If patient already taking oral calcium channel- or beta- blocker, choose same drug group first

- If difficulty achieving adequate rate control, consider using the other first-line agent, IV digoxin, or cardiology consultation

- Calcium channel blocker:

- Avoid if acute heart failure or known LV dysfunction (POCUS may be helpful)

- Diltiazem $0.25 \mathrm{mg} / \mathrm{kg}$ IV over $10 \mathrm{~min}$; repeat q15$20 \mathrm{~min}$ at $0.35 \mathrm{mg} / \mathrm{kg}$ up to 3 doses

- Start 30-60 mg PO within 30 min of effective IV rate control

- Discharge on 30-60 mg QID or Extended Release $120-240 \mathrm{mg}$ once daily

- Beta blocker:

- Metoprolol 2.5-5 mg IV over 2 min, repeat q1520 min up to 3 doses

- Start 25-50 mg PO within 30 min of effective IV rate control

- Discharge on 25-50 mg BID

- Digoxin is second line, as slow onset:

- $\quad 0.25-0.5 \mathrm{mg}$ loading dose, then $0.25 \mathrm{mg}$ IV q4-6 $\mathrm{h}$ to a max of $1.5 \mathrm{mg}$ over $24 \mathrm{~h}$; caution in renal failure

- Consider first line if hypotension or acute HF

- Heart rate target: $<100 \mathrm{bpm}$ at rest, $<110$ walking 


\section{Rhythm control}

- Either pharmacological or electrical cardioversion acceptable, per patient and physician preference:

- Consider previous episodes; if one doesn't work, try the other

- Pre-treatment with rate control agents not recommended - ineffective and delays treatment

- Pharmacological cardioversion:

- Procainamide IV- $15 \mathrm{mg} / \mathrm{kg}$ in $500 \mathrm{ml} \mathrm{NS}$ over $60 \mathrm{~min}$, maximum $1500 \mathrm{mg}$

- Avoid if $\mathrm{SBP}<100 \mathrm{~mm} \mathrm{Hg}$ or QTc $>500 \mathrm{~ms}$

- Interrupt infusion if BP drops or QRS lengthens visibly (e.g., > 30\%)

- Check QTc after conversion

- Amiodarone IV not recommended-slow, low efficacy

- Less commonly used options include: vernakalant IV, ibutilide IV, propafenone PO and flecainide PO

- Electrical cardioversion

- Setup-minimum 2 staff (RN/RRT; RN/RN), $2^{\text {nd }}$ physician ideal

- Procedural sedation per local practice-e.g., Fentanyl, Propofol

- Pad/paddle position - either antero-lateral or anteroposterior acceptable:

- Avoid sternum, breast tissue

- If failure, apply pressure with paddles, try the other position

- Start with 150-200 J synchronized—avoid starting with low energy level

- Many patients can be discharged as soon as $30 \mathrm{~min}$ after conversion if treated with IV procainamide or ECV

\section{Rapid ventricular pre-excitation (WPW)}

- Urgent electrical CV usually required

- Procainamide IV if stable
- AV nodal blocking agents contraindicated: digoxin, calcium channel-, beta-blockers, adenosine, amiodarone

\section{Stroke prevention}

\section{Who requires anticoagulation?}

- Antithrombotic therapy prescribed at discharge is for long-term stroke prevention

- For OAC contraindications see the 'McMaster Checklist'

- If CHADS-65 positive (any of age $\geq 65$, diabetes, hypertension, heart failure, stroke/TIA) initiate OAC prior to discharge; consider shared decision making to include patients' preferences with regards to risks and benefits:

- DOACs preferred over warfarin

- Use warfarin (DOACs contraindicated) if mechanical valve, moderate-severe mitral stenosis, severe renal impairment $(\mathrm{CrCl}<30 \mathrm{ml} / \mathrm{min})$

- If stable CAD, discontinue ASA

- If CAD with other anti-platelets or recent $\mathrm{PCI}<12$ months, consult cardiology

- If CHADS-65 negative, OAC might be considered for a 4-week period after careful consideration of risks and benefits and a shared decision-making process with the patient; ensure patient is aware anticoagulation will be discontinued after 4 weeks

- CHADS-65 negative and stable coronary, aortic, or peripheral vascular disease, ensure patient is on ASA $81 \mathrm{mg}$ daily

- Patients already taking anti-platelet agents require follow-up with cardiology

- If TEE-guided CV, must initiate DOAC immediately $\times 4$ weeks

- If warfarin, need LMW heparin bridging

- Patients who convert spontaneously before ED treatment should generally be prescribed OAC according to the CHADS-65 criteria 


\section{'McMaster Checklist' for safe anticoagulant prescribing}

Absolute contraindications

$\square$ Already on anticoagulant

$\square$ Current serious bleeding

(GI/GU/intracranial/retroperitoneal)

Relative contraindications

$\square$ Prescribed $\mathbf{2}$ antiplatelets

(ASA, clopidogrel, ticagrelor, prasugrel)

$\square$ Recent serious bleeding

(GI/GU/intracranial/retroperitoneal)

$\square$ Cirrhosis of the liver

$\square$ Platelet count $<\mathbf{1 0 0}$

$\square$ Hemoglobin $<80$
Contraindications to DOAC

$\square$ Prescribed carbamazepine, phenytoin, antiHIV, antifungal or antiTB interacting medications

$\square$ Creatinine clearance $<\mathbf{3 0 ~} \mathrm{ml} / \mathrm{min}$

$\square$ Pregnant or breast feeding

\section{DOACs and warfarin}

- See Thrombosis Canada App for details; avoid in pregnancy, breastfeeding

- Consult nephrology or thrombosis if $\mathrm{CrCl}<30 \mathrm{ml} / \mathrm{min}$

- Provincial formularies may require Limited Use codes, e.g. failure of warfarin or INR monitoring not possible:

- Dabigatran-150 mg BID; use $110 \mathrm{mg}$ BID if age $>80$ years, or $>75$ years with bleeding risk

- Rivaroxaban-20 mg daily; use $15 \mathrm{mg}$ daily if $\mathrm{CrCl}$ $30-49 \mathrm{ml} / \mathrm{min}$

- Apixaban-5 mg BID; use $2.5 \mathrm{mg}$ BID if two of: (1) serum creatinine $>133 \mathrm{umol} / \mathrm{L}$, (2) age $>80$ years, or (3) body weight $<60 \mathrm{~kg}$

- Edoxaban-60 mg daily; use $30 \mathrm{mg}$ daily if $\mathrm{CrCl}$ $30-50 \mathrm{ml} / \mathrm{min}$ or weight $<60 \mathrm{~kg}$; important drug interactions

- Warfarin

- Initiate warfarin: $5 \mathrm{mg}$ daily; (1-2 $\mathrm{mg}$ daily if frail, low weight, Asian descent):

- Heparin bridging not required unless TEE-guided CV

- Arrange for INR blood test and review after 3 or 4 doses of warfarin. Subsequent warfarin doses should be communicated to patient on the day of the INR test

\section{Disposition and follow-up}

\section{Admission to hospital}

- Patients rarely require hospital admission for uncomplicated acute AF/AFL unless they:

- Are highly symptomatic despite adequate treatment

- Have ACS with significant chest pain, troponin rise, and ECG changes

- No need to routinely measure troponin, small demand rise expected

- Have acute heart failure not improved with ED treatment

\section{Follow-up issues}

- Recommend physician follow-up $<7$ days if new warfarin or rate control meds

- Recommend cardiology / internal medicine follow-up in 4-6 weeks if not already followed or if new medications prescribed

- Provide handout (available from Thrombosis Canada) describing new medication, atrial fibrillation, and followup; early renal function monitoring if new DOAC

- Do not initiate anti-arrhythmic agents like amiodarone or propafenone in the ED

- If sinus rhythm achieved, generally no need to initiate beta- or calcium channel-blockers 


\section{Box 1. Advisory committee members}

\author{
Academic HSC Emergency Medicine \\ Ian Stiell - Ottawa \\ Frank Scheuermeyer - UBC \\ Suneel Upadhye - McMaster \\ Clare Atzema - Toronto \\ Andrew McRae - Calgary \\ Kerstin deWit - Queen's (Thrombosis) \\ Patrick Archambault - Laval \\ Warren Cheung - Ottawa \\ Debra Eagles - Ottawa \\ Alain Vadeboncoeur - Montreal \\ Ottawa Staff \\ Erica Brown \\ Jennifer Brinkhurst
}

\author{
Community Hospital Emergency Medicine \\ Troy Tebbenham - Peterborough \\ Rick Mann - Mississauga \\ Rupinder Sahsi - Kitchener \\ Geneviève Baril - Granby \\ Cardiology \\ Allan Skanes - Western \\ Paul Angaran - Toronto \\ Marc W. Deyell - UBC \\ Ratika Parkash - Dalhousie \\ Patient \\ Christian Chabot - Quebec City
}

\section{Background and methods}

The 2021 CAEP Acute Atrial Fibrillation/Flutter Best Practices Checklist has been updated from the original version published in 2018 [1]. These checklists have been created to assist emergency physicians in Canada and elsewhere manage patients who present to the emergency department (ED) with acute/recent-onset atrial fibrillation (AF) or flutter (AFL). The checklist focuses on symptomatic patients with acute AF or AFL, i.e. those with recent-onset episodes (either first detected, recurrent paroxysmal or recurrent persistent episodes) where the onset is generally less than 48 $\mathrm{h}$ but may be as much as seven days. These are the most common acute arrhythmia cases requiring care in the ED. Canadian emergency physicians are known for publishing widely on this topic and for managing these patients quickly and efficiently in the ED [2, 3, 4].

The 2018 Checklist project was funded by a research grant from the Cardiac Arrhythmia Network and the resultant guidelines were formally endorsed by the Canadian Association of Emergency Physicians (CAEP). We chose to adapt, for use by emergency physicians, existing high-quality clinical practice guidelines (CPG) previously developed by the Canadian Cardiovascular Society (CCS) [5-7]. These CPGs were developed and revised using a rigorous process that is based on the GRADE (Grading of Recommendations Assessment, Development and Evaluation) system of evaluation [8]. With the assistance of our PhD methodologist (IG), we used the recently developed Canadian CAN-IMPLEMENT $\odot$ process adapted from the ADAPTE Collaboration $[9,10]$. We created an Advisory Committee consisting of ten academic emergency physicians (one also expert in thrombosis medicine), four community emergency physicians, three cardiologists, one $\mathrm{PhD}$ methodologist, and two patients. Our focus was four key elements of ED care: assessment and risk stratification, rhythm and rate control, short-term and longterm stroke prevention, and disposition and follow-up. The advisory committee communicated by face-to-face meetings, teleconferences, and email. The checklist was prepared and revised through a process of feedback and discussion on all issues by all panel members. These revisions went through ten iterations until consensus was achieved. We then circulated the draft checklist for comment to approximately 300 emergency medicine and cardiology colleagues. Finally, the CAEP Standards Committee posted the Checklist online for all CAEP members to provide feedback (Fig. 1).

Early in 2021 the same Checklist Advisory Committee reconvened (with one additional academic cardiologist) to discuss updates based upon new evidence $[3,4,11]$, the 2018 and 2020 CCS guidelines [12, 13], and several commentaries that had expressed the concern of the Canadian ED community $[14,15]$. The Advisory Committee met twice virtually and reached consensus on updates through repeated email exchanges. The panelists then sought further feedback from their own colleagues in emergency medicine and cardiology. Finally, the 2021 Checklist was posted by CAEP for further member feedback prior to final approval. The panel continues to believe that, overall, a strategy of ED cardioversion and discharge home from the ED is preferable from both the patient and the healthcare system perspective, for most patients. Many notable revisions were incorporated, including:

1. The safety of urgent cardioversion for acute AF/AFL depends upon anticoagulation status, prior stroke, valvular heart disease, time since onset, and CHADS criteria. Patients presenting between 12 and $48 \mathrm{~h}$ may only be cardioverted if they have 0 or 1 of the CHADS- 65 crite- 


\section{CAEP AF Best Practices}



Fig. 1 Overview of 2021 CAEP AF/AFL best practices checklist

ria. We found that the CCS reference to $\mathrm{CHADS}_{2}$ Scale problematic as most ED physicians no longer use that scale.

2. Anticoagulation for CHADS-65 positive patients should be initiated in the ED unless there are contradictions as per the "McMaster Checklist" created by Dr. de Wit.

3. We disagree with the CCS suggestion of 4 weeks of anticoagulation for patients who are CHADS-65 negative as this was a weak recommendation per the GRADE system, based upon low quality evidence. We suggest that oral anticoagulation might be considered for a 4-week period after careful consideration of risks and benefits and a shared decision-making process with the patient.

Our hope is that the 2021 CAEP Acute Atrial Fibrillation/Flutter Best Practices Checklist will standardize and improve care of AF and AFL in large and small EDs alike. We believe that these patients can be managed rapidly and safely, with early ED discharge and return to normal activities.
Acknowledgements Funding for this guideline was supported by the Cardiac Arrhythmia Network of Canada (CANet) as part of the Networks of Centres of Excellence (NCE). Dr. Stiell has received unrestricted research support from InCarda Therapeutics and Cipher Pharmaceuticals. Dr. Angaran has received research funding and/ or honoraria from BMS-Pfizer Alliance and Servier, Dr. DeWit has received research funding from Bayer. Dr. Deyell has received honoraria and research funding from Biosense Webster, Bayer, BristolMyers-Squibb, Abbott, and Servier. Dr. Skanes has received honoraria from Boehringer Ingelheim, Bayer, Pfizer, and Servier. Dr. Tebbenham has received honoraria from Cardiome Pharma Corp. We thank the hundreds of Canadian emergency physicians and cardiologists who reviewed the draft guidelines and who provided very helpful feedback.

Open Access This article is licensed under a Creative Commons Attribution 4.0 International License, which permits use, sharing, adaptation, distribution and reproduction in any medium or format, as long as you give appropriate credit to the original author(s) and the source, provide a link to the Creative Commons licence, and indicate if changes were made. The images or other third party material in this article are included in the article's Creative Commons licence, unless indicated otherwise in a credit line to the material. If material is not included in the article's Creative Commons licence and your intended use is not permitted by statutory regulation or exceeds the permitted use, you will need to obtain permission directly from the copyright holder. To view a copy of this licence, visit http://creativecommons.org/licenses/by/4.0/. 


\section{References}

1. Stiell IG, Scheuermeyer FX, Vadeboncoeur A, et al. CAEP acute atrial fibrillation/flutter best practices checklist. Can J Emerg Med. 2018;20(3):334-42.

2. Atzema CL, Jackevicius CA, Chong A, Dorian P, Ivers NM, Parkash R, Austin PC. Prescribing of oral anticoagulants in the emergency department and subsequent long-term use by older adults with atrial fibrillation. CMAJ. 2020;191(49):E1345-54.

3. Scheuermeyer FX, Andolfatto G, Christenson J, et al. A mulitcenter randomized trial to evaluate a chemical-first or electricalfirst cardioversion strategy for patients with uncomplicated acute atrial fibrillation. Acad Emerg Med. 2019;26:969-81.

4. Stiell IG, Sivilotti MLA, Taljaard M, et al. Electrical versus pharmacological cardioversion for emergency department patients with acute atrial fibrillation (RAFF2): a partial factorial randomised trial. Lancet. 2020;395(10221):339-49. https://doi.org/ 10.1016/s0140-6736(19)32994-0.

5. Stiell IG, Macle L. Canadian cardiovascular society atrial fibrillation guidelines 2010: management of recent-onset atrial fibrillation and flutter in the emergency department. Can J Cardiol. 2011;27(1):38-46.

6. Verma A, Cairns JA, Mitchell LB, et al. 2014 focused update of the canadian cardiovascular society guidelines for the management of atrial fibrillation. Can J Cardiol. 2014;30(10):1114-30.

7. Macle L, Cairns J, Leblanc K, et al. 2016 Focused update of the Canadian Cardiovascular Society Guidelines for the management of atrial fibrillation. Can J Cardiol. 2016;32(10):1170-85. https:// doi.org/10.1016/j.cjca.2016.07.591.
8. Guyatt GH, Oxman AD, Vist GE, et al. GRADE: an emerging consensus on rating quality of evidence and strength of recommendations. BMJ. 2008;336(7650):924-6.

9. Collaboration TA. The ADAPTE process: resource toolkit for guideline adaptation, 2009:1-95.

10. Harrison MB, van den Hoek J, Graham ID. CAN-IMPLEMENT: planning for best-practice implementation. Philadelphia, PA, 2014:1-148.

11. Pluymaekers NAHA, Dudink EAMP, Luermans JGLM, et al. Early or delayed cardioversion in recent-onset atrial fibrillation. N Engl J Med. 2019.

12. Andrade JG, Verma A, Mitchell LB, et al. 2018 Focused update of the canadian cardiovascular society guidelines for the management of atrial fibrillation. Can J Cardiol. 2018;34(11):1371-92.

13. Andrade JG, Aguilar M, Atzema C, et al. The 2020 Canadian Cardiovascular Society/Canadian Heart Rhythm Society Comprehensive Guidelines for the management of atrial fibrillation. Can J Cardiol. 2020;36(12):1847-948. https://doi.org/10.1016/j.cjca. 2020.09.001.

14. Stiell IG, McMurtry MS, McRae A, et al. Safe cardioversion for patients with acute-onset atrial fibrillation and flutter: practical concerns and considerations. Can J Cardiol. 2019;35(10):1296300. https://doi.org/10.1016/j.cjca.2019.06.007.

15. Stiell IG, McMurtry MS, McRae A, et al. The Canadian Cardiovascular Society 2018 guideline update for atrial fibrillation-a different perspective. CJEM. 2019;21(5):572-5. https://doi.org/ 10.1017/cem.2019.399. 